\title{
THE NEW EXPERIMENTALISM AND THE VALUE OF EXPERIMENTAL JUSTIFICATION IN EMPIRICAL SCIENCES*
}

\begin{abstract}
This article briefly presents and characterizes a relatively young (nineteen-nineties) trend in methodology, the theory of science - and philosophy, called "the new experimentalism". The fundamental problem is determined by the question about the value of the new experimentalism and experimental grounds of scientific knowledge in empirical sciences. In the first part of the article, the previous (old) experimentalism is presented. First of all, the history of the experimental method is outlined and the definitions of experiment, object, phenomenon, and of the carried out and analyzed observation are provided. It is shown why the main proposition of experimentalists - "determining a fact based on sensory experience" is fallacious. The second part describes the way in which the representatives of the new experimentalism try to identify and characterize those factors of an experiment that guarantee the objectivity of its result; demonstrate that results are not only determined by psychological, historical, sociological or economic factors but also that they exist in nature as real objects and events. A correct and reliable analysis of the experiment and its results may - according to the new experimentalists - contribute to this conclusion. Therefore, the important role and value of the experimental foundation of social activity in general, and in particular, for the natural sciences, is rightly noted.
\end{abstract}

Keywords: new experimentalism; natural sciences; scientific cognition; theory

1. Introduction. 2. The previous ("old") experimentalism. 2.1. Experiment. 2.2. Object - phenomenon - observation. 2.3. "Naive" experimentalism. 2.4. Important facts. 2.5. Rules of the experimental procedure. 2.6. Historical examples. 2.7. Results of an experiment and theory. 2.8. The status of scientific cognition. 3. Objectivity of the results of an experiment according to the new experimentalism. 3.1. Fundamental methodological postulate (point of departure). 3.2. Extension of the postulate. 3.3. The positive function of an unsuccessful experiment.

4. Conclusions: The achievements and perspectives of the new experimentalism.

* This article was originally published in Polish as: M. Bombik, Nowy eksperymentalizm a wartość eksperymentalnego uzasadnienia w naukach empirycznych, Studia Philosophiae Christianae 41(2005)1, 5-40. The translation of the article into English was financed by the Ministry of Science and Higher Education of the Republic of Poland as part of the activities promoting science - Decision No. 676/P-DUN/2019 of 2 April 2019. Translation made by GROY Translations. 


\section{INTRODUCTION}

R. Ackermann is considered to be the founding father of the new trend in the contemporary theory and methodology of the empirical sciences that was started at the beginning of the nineteen nineties ${ }^{1}$. This trend, as I. Hacking emphasizes ${ }^{2}$, attempts to demonstrate that the experiment-based natural theories cannot be entirely reduced, as the opponents of the value of experiment for scientific cognition propose, to a subjective point of view, to psychological, historical or socio-economic determinants. The new experimentalism points to such properties, elements or moments of experimental research that allow considering experimental results as objectively existing facts, and not as just creations determined by a previous adoption of a theory in the framework of which an experiment is conducted and interpreted.

As for the so-called scientific revolutions which, as noted by T. S. Kuhn and the advocates of the trend initiated by him in the methodology and theory of natural sciences, entirely change the previous paradigm of science - which in consequence means advocating the absence of continuity in its advancement - the new experimentalism argues that progress in science is possible primarily due to the constant, gradual expansion and enhancement of scientific cognition. Thus, he refers to the idea of cumulative progress in science - which is strongly criticized by some, and rejected by others.

\section{THE PREVIOUS (“OLD”) EXPERIMENTALISM}

The very name "new experimentalism" suggests that before the emergence of this trend, there must have been some other, "old" experimentalism, which is now being replaced or significantly mod-

1 Cf. R. Ackermann, The New Experimentalism, British Journal of the Philosophy of Science 40(1989), 185-190.

2 I. Hacking, Einfuerung in die Philosophie der Naturwissenschaften, Philipp Reclam, Stuttgard 1996, 10. In this Introductory Topics in the Philosophy of Natural Science Hacking also presents the pioneering achievements of the representatives of the new experimentalism. 
ified by the new variety. This visible opposition of names "forces" one to - even briefly - characterize the fundamental assumptions, concepts and main elements of the historical experimentalism and the method related to it.

\subsection{EXPERIMENT}

From the very beginning of the empirical sciences, experiment was an instance of exceptional methodological importance. Combined with observation and measurement, experiment was the most adequate way of justifying the propositions of these sciences, providing arguments for generalizations made, verifying, or alternatively falsifying hypotheses, or assigning them with a new methodological status, i.e. raising them to the rank of scientific laws. The systematic use of experiment as the fundamental method of research - along with observation and measurement - is one of the most characteristic features of modern natural sciences.

Etymologically, the word experiment is derived from Latin, where experimentum, as dictionaries state, is: an attempt, experience, way of recognizing the truth, long deftness for warfare, the ability, proficiency acquired through experience, result, examination, an object of examination, evidence. The common meaning of experiment is the attempt to implement an idea, especially an innovative one in order to try it out in practice, another term for a search for a new solution through trial.

Based on a number of different terms or definitions of experiment found in the methodological literature, the following description seems to be the most fundamental and at the same time the most universal: Experiment is the artificial creation of objects or phenomena for observation and conducting such an observation. Another general description is: Experiment is any verification of a causal hypothesis by examining contrasting situations in which the factors that are suspected to have infuence are subject to control. A more detailed description, functioning primarily in physics and chemistry, has the following form: Experiment is a procedure which involves changing some factor 
(alternatively some factors) in the examined situation in order to verify the hypothesis concerning the consequences of these changes in conditions in which other factors suspected to have effect are subject to control. Obviously, the greater the extent of such control, the more experimental procedure deserves to be considered as correct ${ }^{3}$.

Taking into account the results of an experimental procedure, the following types of experiment are distinguished: positive experiment, negative experiment, and the so-called experimentum crucis. A positive experiment confirms the formulated hypothesis by increasing its probability, but it never provides a final justification for it. A negative experiment completely disproves the tested hypothesis. Experimentum crucis, or crucial or critical experiment, is to be, according to the idea of F. Bacon, the proponent of this idea, such an experiment, by means of which one of the two competing and heretofore equally acceptable hypotheses, is confirmed and the other one disproved. In the literature, apart from other types of experiments, one can find a distinction of the so-called thought experiment in which changes to the situation are introduced only in thought and the consequences of such changes are predicted. The fundamental difference between a thought experiment and the other three types mentioned above is that the conclusions based on a thought experiment are merely a conjecture leading at the most to the idea of a hypothesis, while in the first three types, the results of a real cognitive procedure prove or disprove a hypothesis ${ }^{4}$.

Experimental methods first emerged and were developed in physics and chemistry, becoming the basis for the advancement of these sciences, then they were passed to all fields of natural sciences, and since the twentieth century, they have also played an increasingly important role in various humanist disciplines, especially in the behavioural sciences. The developing practice of experimental research in individual sciences is accompanied by theoretical and

3 Cf. J. Such, Eksperyment, in: Filozofia a nauka, ed. Z. Cackowski, Ossolineum - PAN, Wrocław - Warszawa - Kraków - Gdańsk - Łódź 1987, 120-122.

4 Ibid, 124-129. 
methodological reflection on the experiment as a scientific method. The first program of extensive use of experiment as an inductive generalization tool was developed in the 16th century by F. Bacon. In the 18th century, J. d'Alembert announced that experiment to be the foundation of cognition in physico-chemical sciences. The role and place of experimentation in the inquiry of the empirical sciences was investigated by J. Herschel, W. Whewell, A. Comte and positivists. In developing his famous methods (canons) of induction, which were to serve as a model of reasoning based on elimination induction and the use of experimentation in a research procedure, J. S. Mill generalized the whole practice of experimental research and methodological issues related to it. Since then, this issue has become the subject of constant inquiry by the methodologists of individual empirical sciences 5 .

Analysis of the content of the above-mentioned fundamental and general definition of experiment: Experiment is the artificial creation of objects or phenomena in order to observe them and to carry out this observation indicates that the definiens of this real definition predicates that the characteristic of an experiment is the "artificial creation of objects or phenomena", without defining more closely how to understand object, phenomenon and observation, treating these expressions as intuitively understandable. The compound name: "artificial production" denotes all those objects and phenomena that do not occur in nature by themselves. In order for them to exist or to appear, a proper interference of a human experimenter is needed. The definition of an experiment formulated in this way is a normal definition and meets the condition of translatability, i.e. the word experiment can be eliminated from every linguistic context containing the word experiment, and replaced with the indicated definiens. In addition, if not only qualitative observation but also quantitative observation, combined with measurement, is involved, then the definition quoted includes, although very generally, the relationship of the experiment with observation and measurement. 


\subsection{OBJECT - PHENOMENON - OBSERVATION}

Semantic problems with the definition of experiment only begin when we want to establish more closely and precisely the meanings of the words that are part of its definiens: object, phenomenon, observation.

In the philosophical and scientific literature, there have been many attempts to answer the question what an object is. These answers are therefore aimed at determining the meaning of the name object. The most general concept of the object is defined by W. Nowicki as follows: "by object we shall mean someone or something that can be thought of, and therefore said or written about, regardless of whether this someone or this something exists or existed in reality, or was only conceived by us" ${ }^{6}$. In the definition of an experiment, it is not so much about the object in general as about the type of objects, namely a concrete (real) object. This is because only a concrete object, as opposed to an abstract object, can be artificially produced, observed and measured, as the definition of experiment postulates.

Although the issue of which objects should be classified as concrete and which as abstract, have been widely considered and discussed in philosophy, no clear-cut conclusions have been reached in this regard. In natural science textbooks and relevant literature on the philosophy of science, one can currently point to three widespread, but not identical, designations of a concrete object. Concrete objects are: (1) physical objects and persons; (2) objects occupying a specific place in space and time; (3) objects occupying a specific place in space and time and characterized by inertia. Comparing the quoted phrases, it is not difficult to notice that the first and the third, as opposed to the second, do not consider physical fields, for example, electromagnetic or gravitational field, as concrete objects. The second phrase considers all events, for example, explosion, solar eclipse, accident, process, fire, etc., to be concrete objects, although it

6 W. Nowicki, Podstawy terminologii, Ossolineum - PAN, Wrocław - Warszawa - Kraków -Gdańsk - Łódź 1986, 20. 
seems right to believe, as Nowicki claims, that e.g. in the case of a house fire, the burning house is a concrete object, while fire is only the state of the house. Nowicki puts forward the following postulate for a demarcation line between concrete objects and abstracts: "we will consider as concrete any object that is either matter (animate or inanimate) or any part or form of it, or a physical field or any part or form of it. We will consider as an abstract, on the other hand, any object thought of by man which is neither matter, nor field, nor any part or form of them"7. This conventional postulate does not resolve in an authoritative and final way the dispute over concretes and abstracts but is only a proposal to facilitate the ordering of the issues in the considerations of scientific terminology.

Phenomenon is a term used in the theory of science and philosophy in many different but related meanings. In the modern theory of science, its fundamental meaning (meaning in the narrower sense) can be defined as follows: we call a phenomenon everything that is the object of sensory or mental perception. This general statement is most often extended by adding that it is any empirical fact that is subject to observation by the available methods and means, or the totality of the characteristics and correlations of the objects under consideration, constituting the starting point of research and scientific cognition, thus formulating a broader meaning of the term. Such meanings of phenomenon occur in the works of, among others, F. Bacon, Galileo Galilei, R. Descartes, G. Leibniz and I. Newton. In the philosophical meaning assigned by I. Kant and spread by Kantianists and neo-Kantianists, a phenomenon is an object of possible experience. Only a phenomenon (phenomena) is (are) accessible to our cognition as opposed to the unknowable "things in themselves" (noumena). Kant's definition of a phenomenon is the foundation of the classical version of phenomenalism, a philosophical trend according to which the scope of human cognition is limited to phenomena that are contrasted with transcendental reality in relation to the subject of cognition. Transcendental reality includes beings that 
exist independently of man and are beyond the limits of his experience. There are three basic proposals (and many modifications of these proposals) for solving the problem of being which is different from phenomena, put forward by representatives of different versions of phenomenalism: (1) rejecting the existence of this kind of being; (2) recognizing its existence while stating that it is unknowable; (3) considering the question about the existence of being which is different from phenomena as unresolvable ${ }^{8}$.

Observation in a popular-psychological sense is: perceiving objects or phenomena in order to reach an answer to the question posed. Thus, observation differs from simple perception in that with this second type of cognitive activity we do not ask the question that we want to answer. The basic methodological meaning of the term observation is obtained by limiting the scope of the name perception, emphasizing that it is not about whatever perception, but only about perception which is systematic and planned. Thus: Observation is the planned and systematic perception of objects or phenomena in order to reach an answer to the question posed. Observation in this sense is one of the essential methods of research in the natural sciences. Observation is often put in opposition to experimentation. In observation, the researcher limits himself to observing what is happening without his interference, and in the experiment, he changes or creates new conditions of the examined phenomenon, although, in the practice of scientific inquiry, the borderline between the two methods cannot be determined precisely.

The method of observation is determined by the type of the object or phenomenon being examined and the situation in which the observation is carried out. However, three basic features of correct observation can be pointed out - planning, regularity and selectivity. Planning concerns the order of observation, regularity is about certain intervals, and selectivity is the selection of those facts and characteristics of the objects that are important for solving the cog-

8 More about the phenomenon, cf., among others: M. Hempoliński, Empiryzm, in: Filozofia a nauka, op. cit., 150-150; Z. Cackowski, Fenomenalizm, in: Ibid, 161-169. 
nitive problem. The correctness of the course of observation and the accuracy of its results depend primarily on: (1) quality of the observed data - e.g. their structure, degree of complexity, dynamics of development, etc.; (2) conditions in which the observation is carried out - e.g. distance, lighting, interfering factors, etc.; (3) the observer - his/her motivation, mental characteristics, knowledge of the observed material, individual interpretations made during the observation process, etc. The accuracy and scientific significance of the observation results, in turn, depends on the instruments available to the observer, on the way of recording and interpreting their indications. The ways of recording information obtained through observation are becoming increasingly complex with the development of science. In natural sciences, increasingly objective techniques are used to record the observed material, e.g. film, tape recorder, oscillograph, electroencephalograph, etc., as well as instruments increasing the limited possibilities of cognitive receptors, e.g. microscope, telescope, various types of amplifiers. Convenient conditions for increasing the objectivity of observations are provided by the possibility of multiple repetitions of observations by different researchers, at different times, to verify the hypotheses proposed based on observations made in experimental studies and to check by re-observation the validity of the conclusions drawn from them. However, in no science can the influence of the individual characteristics of an observer on the course of observation be completely eliminated. Thus, the main methodological problem when using observation as a research method in science comes down to attempts to ensure the objectivity of methodological observation and its results 9 .

\section{3. "NAIVE" EXPERIMENTALISM}

On the basis of intuitive and uncritically assumed concepts of $e x-$ periment, experience, object, observation, measurement, a methodo-

9 About observation, its types, the difference between observation and experimentation, cf., among others, Z. Cackowski, Obserwacja, in: Filozofia a nauka, op. cit., 433-444. 
logical trend called experimentalism forms in the theory of empirical sciences which maintains that on the basis of the testimony of our senses, and above all through observation, empirical facts are established, on the basis of which scientific cognition, that is, "knowledge based on facts" is built. In light of the accusations made against this methodological trend, primarily by psychologists, historians, sociologists and theorists of science, one should probably speak of "naive" experimentalism here. Experimentalism referred to the views of modern methodologists: Bacon, Herschel, Whewell, Mill, and it updated and modified these views as research methods in natural sciences improved, to reach its apogee in the period of neopositivism, or logical empiricism, that is, in the nineteen-thirties and nineteen-forties. One of the basic points of the neo-positivist program was the postulate that all knowledge of the world must be based on experience. Of course, the level of "naivety" of 20th-century experimentalism was lower than in the 18th or 19th century, but its methodological correctness, as critics have demonstrated, left a lot to be desired.

Moreover, the experimentalists were aware of the imperfection of human senses in terms of cognition as indicated by the shortcomings and fallacies of numerous observation results, but nevertheless, numerous correct observation results, e.g. the reading of indications of measuring instruments or counting of voice signals of various types of meters, were supposed to justify their position that facts are established based on the testimony of the senses. A critical analysis of the said position leads to the conclusion that the provided verbalization of it is at least a great simplification if there is any degree of its acceptance at all. First of all, it should be noted that natural sciences are not about whatever facts, but only about important and relevant facts. This conclusion raises the question - which facts are relevant to science. The answer to this question depends on the level of theoretical development of a given discipline. 


\subsection{SIGNIFICANT FACTS}

In the world around us, there are many different processes that often overlap, condition or integrate with one another in a complex way. For example, a leaf falling from a tree is subject, as any material body, to the law of gravity, but the place of its contact with the ground is also determined by the resistance of the air, the force of the wind, the state of the rotting process to which it is subject, etc. An exact description of these processes is not possible with even the most meticulous observation. Observations of leaves falling from trees will therefore not confirm Galileo's theory of free-falling objects. This simple example teaches us that in order to obtain facts that are significant for the identification and characterization of processes that are important in nature, facts that constitute the fundament of the natural sciences, it is often not enough to make a simple observation but the intentional intervention of the observer is necessary, consisting of, broadly speaking, isolating the examined process and eliminating the effects of other processes co-occurring with the examined one, it is therefore necessary to conduct an experiment. Although this position seemed trivially obvious from the beginning of conscious use of the empirical method in science, it was only in recent decades that theorists and philosophers of science have undertaken a relevant inquiry into the nature and role of experimentation in science ${ }^{10}$.

Obtaining important (significant) experimental results in a given field is, as the history of experimental research shows, not an easy undertaking. It often took months and years before a significant experiment could be conducted. An account of the enormity of theoretical and practical difficulties that an experimental physicist has to overcome is provided by Chalmers who describes his own experiment from the 1960s, which was intended to provide new information about the energy level of molecules. The experiment involved releasing electrons with a low energy potential from the

10 Cf. A. F. Chalmers, Wege der Wissernschaft (Einfuerung in die Wissenschaftstheorie), Springer - Verlag, Berlin - Heidelberg 2001, 25-26. 
molecules and calculating the amount of energy that electrons lose during this process. A detailed analysis of the conducted experiment leads to the following findings, which the author generalizes to all experimental procedures of this kind.

\subsection{RULES OF THE EXPERIMENTAL PROCEDURE}

(1) Experimental results which are intended to be the fundament of a given science, cannot be obtained only from simple sensory perception. They are the result of properly planned and precisely organized action and their confirmation is largely based on the knowledge and practical skills of the experimenters, which in turn depend on the state of current technological capabilities and the scale of errors always inherent in such procedures.

(2) The assessment of the scientific and practical value of experimental results is also not easy. It is considered pertinent only if the results can be interpreted as confirming the solution to the problem formulated if the experiment was theoretically well prepared, its course was subject to appropriate control and factors whose presence may have distorted the obtained results were eliminated.

(3) Ensuring appropriate conditions for the proper course of an experiment and elimination of elements interfering with this process or distorting its results are possible based on appropriate knowledge in this regard. This knowledge is to indicate what kind of disturbances can occur and how they can be eliminated. Thus, there is a significant relationship between the experimentally established facts and the theory on which the experiment is based. If this knowledge is insufficient, for example, if it is insufficient or fallacious, then the facts obtained are simply pseudo-facts. A consequence of this interconnection between experimental results and the knowledge within which the experiment was carried out - which is always only probable - is that also the results can never be taken as absolutely certain.

(4) Experimental results may become obsolete due to: (a) the emergence of new, better, more precise technologies; (b) the de- 
velopment of knowledge in the light of which the original idea of the construction and organization of the experiment has changed fundamentally or has been significantly modified; (c) theoretical change in the original qualification of the obtained results of an experiment - it turned out that the established facts are not relevant or as momentous as it was previously thought ${ }^{11}$.

\subsection{HISTORICAL EXAMPLES}

These findings are, according to Chalmers, strongly confirmed by the history of the development of the experimental method ${ }^{12}$. The German physicist H. Hertz conducted a series of experiments in the 1980s to learn about the nature of cathode rays. This is the kind of light that appears inside a glass tube, filled with a gas of not very high pressure, in the form of a "thread of light" connecting the anode with the cathode. The result of these experiments was the conclusion that cathode rays are not a stream of electrically charged particles. In support of his position, Hertz points out that the rays under investigation do not deflect when an electric field directed perpendicularly to the direction of their propagation acts on them, although this is how particles with an electric charge should behave. The conclusion of Hertz's experiment today is considered false, and his experiments are considered to be incorrect. Twenty years later, Thomson's research has shown that cathode rays manifest exactly the same deflection in the electromagnetic field as streams of electrically charged particles, and Thomson has measured the ratio between charge and mass of the particles. What enabled Thomson to reject the of Hertz's experimental results was: (1) improved technology of experimentation; and (2) a better understanding of the complexities of the various processes involved in the whole phenomenon.

Electrons of which of cathode rays consist, can ionize the gas molecules in the tube, i.e., deprive them of one or more electrons

11 Ibid, 26-28.

12 About the changes in the understanding of the empirical foundation of science and the development of the experimental method based on the analysis of historical examples, cf. Ibid, 28-32. 
and thus cause them to convert from electrically neutral to positively charged molecules. The ions formed in this way can be accumulated on the metal plates of the equipment and during the experiment, they can produce small additional electric fields. It is likely that these fields prevented Hertz from obtaining the deflection of the cathode rays, which Thomson not only obtained but also measured the angle of their deflection, depending on the charge and mass of electrically charged particles. The improvement of the methodology of conducting the experiment consisted primarily in extending the operating time of special gas suction pumps (the pumps worked for many days), subjecting the entire equipment to long heating at high temperatures and thus removing the remaining gas adhering to the pipe surface in some places and using better-quality electrodes. However, false conclusions of Hertz's experiment do not undermine his authority as one of the best experimenters of that time. Based on his theoretical knowledge and the technical solutions at his disposal, the results of his experiment were correct. And the theoretical and technological modifications or advancement of knowledge, changing the evaluation of previously obtained experimental results, are unpredictable.

Another example is the generation by Hertz of radio waves in 1888. Radio waves, which were a new kind of phenomena in nature, could still be evoked and studied, moreover, they had this scientifically significant consequence that they confirmed C. Maxwell's electromagnetic theory, formulated in the mid-1860s, from which they could theoretically be derived. Most of Hertz's experimental results in this field have survived the test of time and are also of great importance today. However, some of them as well as their interpretations had to be modified, changed or rejected. These facts are examples of the fact that experimental results have to be constantly monitored, checked and improved.

Hertz was able, thanks to his equipment, to produce standing radio waves and measure their length and propagation rate. The results of his experiments indicated that radio waves of greater length propagate faster in the air than in wires, and faster in relation to 
light, while Maxwell's theory predicted that their propagation rate should be equal to that of light, regardless of what environment they propagate in, whether in the air or a wire. The reason for the incorrect measurement was the inappropriate conditions under which the measurement was taken, which, in any case, was presumed by Hertz. Long radio waves bounced off the walls of the laboratory where they were measured, they superimposed, and this led to serious measurement errors. In these conditions, only shorter waves could be measured. The experiment and the measurement of long radio waves a few years later, under better and appropriate conditions, confirmed the theoretical predictions that the speed of propagation of waves is equal to the speed of light.

Problems related to the measurement of radio wavelengths teach us that the results of experiments should not only be an adequate description of what has been discovered but should also be significant from a theoretical point of view, i.e. that they should answer questions that are theoretically important in a given field. And the assessment of when a question is important and to what extent a specific experimental procedure can be the right way to obtain the correct answer depends primarily on the theoretical ideas and practical possibilities of their implementation. The existence of competing theories pertaining to electromagnetic phenomena and predictions formulated on the basis of one of them, namely Maxwell's theory that radio waves should propagate at a speed equal to the speed of light, made Hertz's attempts to measure the speed of radio waves particularly momentous. The understanding of the phenomenon of the reflection of waves led to a proper evaluation of the conditions in which the experiment was conducted. The relatively small space limited by the walls of a laboratory which reflected the waves was unsuitable for this kind of measurements. A change in the measurement conditions soon yielded correct results. The rejection of the results of radio waveform propagation rate measurement conducted by Hertz has nothing to do with the problem of human perception accuracy. Hertz closely observed the course of the experiment, controlled and recorded the phenomena taking place, recorded the indications of the instruments. 
His findings are objective in the sense that anyone who would like to repeat the procedure used by him, will obtain similar results. Thus, the problems related to the findings of Hertz's experiment cannot be reduced to the inaccuracy of observation or the impossibility of repeating the experiment, but to the improper organization of the experimental procedure. No observations, also if they were even more careful, could replace the necessary condition for the success of the experiment in this case, which was a larger space for the propagation of the measured waves than the laboratory area that Hertz had at his disposal for the measurements.

\subsection{RESULTS OF AN EXPERIMENT AND THEORY}

The described examples aptly illustrate how much the acceptance of experimental results depends on the theory within which the experiment is carried out and how radically their evaluations can change due to the development of scientific cognition. A good illustration of the indicated state of affairs may be the observation concerning the increase in the value of the significance of radio waves for scientific cognition since their discovery by Hertz. At that time, one of the many electromagnetic theories was the theory proposed by J. C. Maxwell, who elaborated on the basic ideas of M. Faraday and understood electrical and magnetic phenomena as mechanical states of a substance called ether, permeating everything in the world. This theory assumed - unlike the theories competing with it, claiming that electrical and magnetic phenomena interact from a distance without the mediation of ether - that radio waves travel at the speed of light. The results of Hertz's experimental research, and especially the possibility of generating electromagnetic waves, which is of lasting importance in the development of physics, could be interpreted by him and his contemporaries as evidence of the existence of ether. Twenty years later, in the light of Einstein's theory of relativity, the ether hypothesis was rejected. Still, however, these results are considered to confirm the revised form of Maxwell's theory, which gives up accepting the ether hypothesis and treats electric and magnetic fields as independent phenomena (beings). 
Another example, convincingly illustrating the dependence of experimental results and their interpretation on the theoretical context in which they arise, are molecular weight measurements carried out by chemists in the second half of the 19th century. In the light of the atomic theory of chemical bonds, the chemists of the time assign fundamental importance to the molecular weight measurement. This mainly concerns theories that favoured Proust's hypothesis that the hydrogen atom is the basic element from which other atoms are built. This conviction allowed to expect that the molecular weights, calculated in relation to the hydrogen atom, are expressed as integers. However, accurate measurements of molecular masses carried out by leading 19th-century chemists proved to be worthless in the light of theoretical chemistry when it was discovered that the basic elements of matter occurring in nature are a mixture of isotopes, and knowledge of their mutual weight proportion is of no theoretical significance. F. Soddy, in his brief commentary on this episode of the development of chemistry, compares the fate of a group of outstanding chemists of the 19th century to a tragedy. Their achievements, which were considered by our contemporaries, not without reason, as the peak of precision measurements which were extremely time-consuming and hard-fought with enormous work, turned out - at least from the present perspective - to be as uninteresting and meaningless as, for example, determining the average weight of a collection of bottles, some of which are completely and others only partially filled with liquid.

In this case, the experimental results were also rejected not because of inaccuracies or observation errors as such, i.e. not because of a lack of objectivity of cognition. These results were considered to be "the pinnacle of scientific measurement precision" and undoubtedly modern chemists would have obtained similar results if they wanted to repeat those procedures. The correctness of the experimental procedure for the scientific meaning of the experiment, the scientific applicability of the obtained results is a necessary but not sufficient condition. The above-mentioned examples clearly characterize the properties and characteristics of the experimental procedure and its results, which 
can provide the basis and starting point for scientific cognition in physics, chemistry and other empirical disciplines. Experiments must always be based on the results of the latest experiments. Obsolete results must be constantly modified, changed, rejected as inadequate or fallacious and replaced by better ones. Modification or rejection of the findings of previous experiments can occur for at least four reasons: (1) the sources of possible interference and the irregularities of the experimental process were not eliminated to a sufficient extent; (2) the measurements were based on imprecise or outdated methods; (3) it was noticed that the conducted experiment did not lead to the solution of the problem posed; (4) the problem which the experiment solved has lost its importance - it has become irrelevant. Although these four methodological postulates more or less intuitively guide everyday experimental practice, they have been, and are, weakened or even abolished by certain philosophical assumptions, especially those that state that experimental results, which are the foundation of the cognition of empirical sciences, can and must be unquestionably certain. Moreover, a detailed analysis of the provided examples shows that the relative cognitive status of any experimental results has nothing to do with the psychological issues of human perception ${ }^{13}$.

\subsection{THE STATUS OF SCIENTIFIC COGNITION}

Establishing that experimental results are not simply given with absolute certainty, that they are dependent on the theory that they are supposed to verify, that they are often burdened with errors and

13 The following source material was the basis on which Chalmers based his analysis of historical examples: H. Hertz, Gesammelte Werke, vol. II: Untersuchungen ueber die Ausbreitung der elektrischen Kraft, Bahrt, Leipzig 1894; W. Thomson, P. G. Tait, Handbuch der theoretischen Physik, Vieweg, Braunschweig 1879; J. C. Maxwell, The Kinetic Theory of Gases, Nature 16(1877), 245-246; Idem, Illustrations of the Dynamical Theory of Gases, in: The Scientific Papers of James Clerk Maxwell, 2 volumes, ed. W. D. Niven, Dover, New York 1965; I. Lakatos, Falsifikation und die Methodologie wissenschaftlicher Forschungsprogramme, in: Kritik und Erkenntnisfortschritt, ed. I. Lakatos, A. Musgrave, Vieweg, Wiesbaden 1974. 
therefore require constant verification, poses a serious challenge to the belief that scientific cognition has a special status because it is based in a convincing way on experience. If it is true that the experimental foundation of science is, as shown, cognitively imperfect to such an extent, then the knowledge based on experience will be burdened with errors and flawed at least to the same extent, and will therefore require constant verification. Besides, the establishment of the cognitive status of an experiment in a scientific procedure complicates the allegation of a vicious circle in argumentation, which in this case is a circular form of semantic petitio principii. If the evaluation of the accuracy, correctness and appropriateness of the course of the experiment and its results is carried out within the framework of a given theory, and at the same time these results are to be a confirmation of this theory, then the existence of the vicious circle seems obvious. Science does not seem to be able to work out experimental criteria for determining which of two or more competing theories is true. Often the same experimental results are invoked by representatives of competing theories, interpreting them accordingly. Thus, in the first place, the fundamental question that arises is whether the indicated petitio principii can be overcome in an experimental justification.

An illustration of circular experimental justification can be an experiment conducted by a group of physics students as part of practical classes under the direction of Chalmers. The experiment was to demonstrate that the number of revolutions of an electric coil placed between the poles of a horseshoe magnet is directly proportional to the intensity of the current flowing through the coil in a given time. The experiment confirmed this hypothesis, but a thorough analysis of the whole procedure showed that the confirming result was unconsciously assumed in the construction of the equipment used. The example shows that a vicious circle can appear in the reasoning based on an experiment, but at the same time it indicates that not every experiment has to be burdened with such an error. In general, it can be stated: any experimental procedure is undertaken in order to confirm some hypothesis or theory which is obtained by correctly reading and interpreting the indications of the relevant instruments; however, the theories being tested cannot be identical 
to those underlying the construction of the experimental tools. The indicated postulate outlines the framework preliminary conditions for designing and organizing any experimental research.

Another position concerning the dependence of experimental results on the theories they confirm is as follows: no matter how strongly and to what extent the experiment is controlled by the theory being tested, there are always certain factors to support the view that the results of an experiment are not only determined by the theory being tested but are also determined by the non-theoretical elements of the actual reality. If the experimental equipment has been constructed, for example, the switch lever of the built-in electrical circuit breaker has been closed, a signal will appear on the screen or will not appear, the beam will be deflected or not, the ammeter pointer will move or will not react. One cannot, therefore, "make" the results correspond to theories. The real structure of the world, the physical properties of nature made the deflection of cathode rays not appear in Hertz's experiments, while this phenomenon occurred in Thomson's modified experiment. It was the differences in the construction (organization) of the experiments of both physicists that led to these experimentally different results, not the differences in the theories on which the experiments were based.

The fundamental thesis of the presented position can be formulated as follows: because the results of experiments are more determined by the elements of the real world than by theoretical structures, these results may be criteria for the truthfulness of theory. However, this does not mean that scientifically valuable results of experiments are readily available, that they are not burdened with errors, that their usefulness and rank are simply given and immediately visible. Nevertheless, the effort to properly and reliably test scientific theories through experimentation is a rational and relevant undertaking; moreover, the history of science provides many examples where this was made complete with what today is called scientific discoveries or successes ${ }^{14}$.

14 Two fundamental positions on the interpretation of the relationships between theory and experimentation results, cf. A. F. Chalmers, Wege der Wissernschaft, op. cit., 33-34. 


\section{OBJECTIVITY OF THE RESULTS OF AN EXPERIMENT ACCORDING TO THE NEW EXPERIMENTALISM}

What the representatives of the new experimentalism are trying to do is identify, describe and characterize those elements of the experimental procedure that ensure the objectivity of experimental results, i.e. decide that these results cannot be interpreted solely in subjective categories as the results of psychological, historical, sociological and economic conditions, but that they are the result of interactions taking place in the existing natural world. And as Chalmers shows ${ }^{15}$, the prototype of the electric motor constructed by Faraday and the emergence of new, theoretically neutral electromagnetic phenomena associated with its functioning posed a great challenge for the then young science of electricity. The existing electromagnetic theories tried to describe and explain these phenomena. Discrediting the value of Faraday's experimental results by stating that all experiments are flawed can be easily overruled. Faraday described the experimental device in detail and attached to the description instructions, according to which anyone - especially his contemporary theoretical opponents - was able to construct a similarly functioning version of the electric motor. Some unsuccessful attempts were neither a surprise nor something important, as they were the result of insufficiently strict observance of the instructions. And although the theoretical explanation of engine operation, accepted today, differs significantly from the explanations given by Faraday and Ampere, it is true that under normal conditions, i.e. the conditions described in the original instruction, the engine will work also today. It also seems unlikely that future advances in the development of electromagnetic theories will cause the engine to stop working. On the grounds of the above, it can therefore be argued that such phenomena produced as a result of controlled experiments are not the result of cognitive errors and that they really and permanently exist in the natural world. If scientific progress is understood as an accumulation of such permanent 
phenomena, it is achieved on this path regardless of the different theories, interpretations and meanings of the concept.

The second example strengthening the position of the representatives of new experimentalism can be, for example, certain episodes from Hertz's biography, described by J. Buchwald ${ }^{16}$ in his detailed study of his scientific career. Hertz's ambition, in a certain period of his life, was an explicit "hunt" for new experimental discoveries. However, some of his "discoveries" in the field of electromagnetism did not enjoy widespread acceptance. This was because, as the representative of the new experimentalism argues, Hertz was a supporter of Helmholtz's electromagnetic theory, which was then one of many existing theories (e.g. Weber's theory, Maxwell's theory) and the results of his experimental research could be assessed and justified in the light of the acceptance of Helmholtz's entire theory, i.e., they were not objectively existing phenomena of the real world, but only artefacts produced by the theory. The situation has changed radically since Hertz began to experimentally generate radio waves. Their real existence could be demonstrated regardless of any theory on which the experimental device which produced them in a controllable way was based. Therefore, according to the new experimentalists, the objectivity of the obtained results of the experiment is supported by their controllable production, and their description and evaluation independent of any theory.

How, for example, when observing through a microscope, we can determine whether we are dealing with an observed real object or just an artefact, is convincingly illustrated by Hacking ${ }^{17}$. A grid of small squares was engraved on glass, and then it was reduced photographically to such an extent that it is no longer visible to the naked eye. When observed under an electronic microscope, it becomes clear and legible. This, as emphasized by Hacking, is a decisive argument for the fact that microscopic magnification is au-

16 J. Buchwald, The Creation of Scientific Effects, University of Chicago Press, Chicago 1989.

17 I. Haking, Einfuerung in die Philosophie der Naturwissenschaften, op. cit., 309ff. 
thentic (it is not a delusion) and that it is independent of the theory of microscope construction. A biologist now observes, for example, red blood cells placed in an experimentally appropriate way on the grid. He sees certain bodies with a relatively high density inside the cell and asks himself whether these bodies are really blood elements or artefacts produced by the microscope. He initially assumes that they are structures artificially created by the microscope, marking accordingly the cells in which they appeared. He then observes the examined biological material through a fluorescence microscope, i.e. a microscope operating based on completely different principles than the electron microscope. The image is identical in the sense that the same objects appeared in the same places in the grid. The comparative analysis of the images obtained in this way is a sufficient and sufficiently strong argument for the conviction that the objects observed are blood cells rather than artefacts. The possibility for microscopes built on theoretically different principles to produce identical "facts" is, as Hacking stresses, most unlikely. The reality of the existence of the object observed with a microscope is supported by the fact that when adopting this position one does not have to refer to the knowledge concerning the functioning of these research instruments or to the theory of their construction.

\subsection{FUNDAMENTAL METHODOLOGICAL POSTULATE}

Deborah Mayo ${ }^{18}$ is one of the philosophers of science in general, and theoretically leading representatives of new experimentalism. She tries to describe, extremely rigorously in methodological terms, the way in which a statement justified by an experiment (experience) can be considered credible. The general postulate, which is the foundation and at the same time the starting point of her considerations, can be formulated as follows: a statement (proposition) can only be considered as experience-based if the various (possibly all) possibilities

18 D. Mayo, Error and the Growth of Experimental Knowledge, University of Chicago Press, Chicago 1996. 
of overturning (falsifying) it are examined and eliminated. In other words: a certain statement can only be claimed to be derived from experience (derived experimentally) if it has undergone a thorough revision in the course of an experimental procedure, in the sense that its acceptance would be impossible if the statement was false. The following research situation may illustrate this kind of revision. Let us suppose that the re-check of Snell's refraction law showed that more accurate measurements of the angles of incidence and refraction of the light ray showed a certain range of possible measurement errors. Let us further suppose that the measurements burdened with these errors still confirm this law. Mayo asks whether this revision of the measurements could have resulted in the claim that this law is experimentally confirmed. And she answers in the negative, arguing that because of the measurement inaccuracies (errors), the law would pass the experimental revision even if it were false, and another law that would not be much different from Snell's law, would be true.

Mayo's position is illustrated by Chalmers ${ }^{19}$ who uses experiments conducted by students as part of practical classes conducted by him. The students were to perform a series of not very accurate measurements concerning the re-confirmation of Snell's law. Then they were to check some formulations of the refraction law from the antiquity and the Middle Ages, which were alternative to Snell's law. It turned out that these alternative "laws" passed the measurement test as a result of a too wide range of systematic measurement errors associated with this method of measurement. The illustration clearly shows that the students' experiments did not meet the conditions for a thorough revision of Snell's law. This law would pass experimental measurements even if it was false; moreover, the historical alternatives turned out to be "true".

Another illustration of Mayo's methodological position may be the analysis of the following situation. In the morning I drank two cups of strong coffee and in the afternoon I felt a distinct headache. Has the sentence "Morning coffee caused my headache" been (experimentally) confirmed by this observation? The answer to this

19 A. F. Chalmers, Wege der Wissernschaft, op. cit., 159. 
question, according to Mayo, is obviously “No!". A positive response would require the elimination of all other - in this case very different - causes that might have caused my headaches. If there is a real causal link between coffee drinking and my headache, the controllable tests (experiments) would have to eliminate all other possible causes of this state of affairs. The experiment, therefore, confirms the statement only if other possible ways of confirming it did not occur (were eliminated) and it would be most unlikely for the statement that passed the experimental test not to be true.

\subsection{EXTENSION OF THE METHODOLOGICAL POSTULATE}

Mayo's methodological position on the assessment of the cognitive value of an experiment in the justification of propositions, statements and, above all, theories, formulated within the natural sciences, is to be broadened and deepened first of all by the cognitive analysis of the socalled "tacking-paradox". Let us assume that Newton's theory T has been confirmed through careful observation of a comet's movement. Care was taken to eliminate situations that could lead to observation errors, such as gravity forces of the nearby planets, the slowing down of the comet's movement as a result of the resistance of the Earth's atmosphere through which the comet passes, etc. Let us now construct theory T' in such a way that we add the proposition "Emeralds are green" to Newton's theory T and ask: can theory T' be confirmed by observation? If we assume that a certain prognostic proposition $\mathrm{p}$ confirms a theory when it is a consequence of this theory and is experimentally positively verified, then theory $\mathrm{T}^{\prime}$ is, contrary to our intuition, confirmed by observations from which it can be concluded that the proposition "Emeralds are green" is true. All the theories constructed similarly to theory T' will be confirmed in this way. According to Mayo, however, theory $\mathrm{T}$ ' is not actually confirmed and the paradox has been removed. Mayo argues as follows: based on the assumption of eliminating all possible sources of error, it can be argued that the actual trajectory of the comet could not have been compatible with Newton's theory if that theory had not been true. The truthful- 
ness of theory T' cannot be argued in this way because the probability that the comet's trajectory will correspond to Newton's predictions will remain unchanged if, for example, some emeralds were blue; and then theory T' would be false. Theory T' cannot be confirmed by this dubious experiment because various possibilities that could falsify the proposition "emeralds are green" have not been explored. Observations of the comet are a strong test for theory T, but not for theory $\mathrm{T}$ '.

Using similar reasoning, Mayo reviews the theoretical considerations leading to conclusions that transcend the boundaries of experimental research. Specifically, this pertains to the revision of A. Einstein's predictions about the behaviour of a light beam in the gravitational field which was carried out by A. Eddington. Eddington used a solar eclipse to check the relative position of stars when their light on its way to Earth was passing near the Sun and compared it with the positions that could be determined when the stars were at a considerable distance from the Sun. The differences were visible. A detailed analysis of the experiment also called the "solar eclipse" experiment, led Mayo to the conclusion that Einstein's law of gravitation, which can be derived from the general theory of relativity, is confirmed by the experiment, while the general theory of relativity is not. In support of this conclusion, Mayo uses the following argument: if one assumes that the results of the solar eclipse experiment strengthen the general theory of relativity, it is necessary to show that obtaining these results would be most improbable if the general theory of relativity was false. It must therefore be possible to eliminate false relationships between the theory and the results obtained. This is impossible in this case because there is a class of theories trying to describe the correlations between time and space and they all assume the existence of Einstein's law of gravitation and thus the phenomenon related to the solar eclipse. Thus, if any of the theories alternative to Einstein's theory were true, the same results would be predicted for the eclipse experiment. Consequently, these results do not constitute an experimental test for the general theory of relativity, since they are unable to resolve the alternative between it and other existing theories. The claim that the solar eclipse experiment confirms the general theory of relativity exceeds experimental results and thus is not justified. 
The situation changes when one takes into account a claim limited as to the scope, i.e. the indicated law of gravitation, which is already confirmed by the results of the eclipse experiment. However, before these observations can be considered to justify this theory, other possible causes leading to the same observational results must be eliminated. Only then can it be concluded that the observed changes in the relative positions of stars only occur when Einstein's law of gravitation applies. Mayo proceeds to demonstrate in detail how alternative formulations in relation to Einstein's theory can be rejected on the basis of such reasoning. For example, the classical Newtonian alternative, based on the assumption that gravitation is inversely proportional to the square of the distance between the photons and the Sun and assuming that photons have mass. Einstein's law of gravitation has been subject to strong verification on the basis of the eclipse experiment - negative results would lead not only to its rejection but also to the rejection of the general theory of relativity, since the falsification of the consequences of this theory, which the law represents, would also be a falsification of the whole theory.

The new experimentalists, generally speaking, are looking for ways to confirm the truthfulness of the cognition gained in science and often entangled in very complex and complicated theories. Mayo's research work harmonizes well with this aspiration. According to this position, as demonstrated, experimental generalizations can be strongly verified. The increment of scientific cognition (scientific advancement) is understood by the representatives of the new experimentalism as the accumulation (aggregation) and an increase in the number of such generalizations (laws) ${ }^{20}$.

\subsection{THE POSITIVE FUNCTION OF AN UNSUCCESSFUL EXPERIMENT}

Experimental results, according to the new experimentalism, confirm true statements, because if a given statement is false, certain

20 For more on the analyses carried out and conclusions reached by Mayo, cf. A. F. Chalmers, Wege der Wissernschaft, op. cit., 159-162. 
experimental results would be most unlikely to obtain. In addition, Mayo emphasizes and analyses the positive cognitive function of an experiment which is unsuccessful or was conducted in an incorrect way, stating that the experiment teaches "learning from mistakes". The experiment, therefore, plays a double role in this approach. It is used to detect an error in a previously accepted proposition, i.e. it serves to falsify a claim, but at the same time, it determines certain previously unknown phenomena (positive role). This positive role of experiments leading to wrong results is illustrated by Mayo's modification of Kuhn's concept of "normal science". When asked why astrology was not classified as a scientific discipline, Popper answers: because its claims are not falsifiable. Kuhn, on the other hand, believes that astrology was and is falsifiable; in the 16th and 17th centuries, when astrology was acceptable, astrologers made verifiable predictions, many of which proved to be false. Today, on the basis of scientific theories, one can also make predictions, and some predictions turn out to be false. The difference between astrology and scientific theories is, according to Kuhn, the fact that science can "learn" from falsification, while astrology cannot. In science, there is a tradition of "solving puzzles", and this tradition is lacking in astrology. Science can do more than just falsify, it can also "overcome falsifications", that is, replace falsified propositions with other, cognitively valuable ones. In this perspective, one can speak of a kind of irony with regard to Popper, who captured his contribution to science with words: "we learn by our mistakes". His methodological programme failed, however, because he was satisfied with finding errors, falsifying, and was unable to complement this negative aspect of scientific procedure with a positive aspect, i.e. he did not teach how to overcome errors - falsifications.

Mayo advocates Kuhn's methodological program of understanding and practicing science, putting a sign of equality between his "normal science" and experiment-based science, pointing, for example, to two episodes from the history of science that illustrate the positive impact of detected errors on the further development of scientific cognition. First, he refers to the commonly known difficulties that arose in the 
mid-nineteenth century before Newton's theory which described the movements of planets orbiting the Sun as a result of the observed anomalies in the orbit of Uranus. The positive side of the problem was, says Mayo, the discovery of the causes of these interpretative difficulties, which, as we know, led to the discovery the planet Neptune which was not known before. The second example is Hertz's experimental work on cathode rays which led him to the conclusion that these rays are not deflected when they are exposed to an electric field. The error of this conclusion was shown in an experiment - as has already been mentioned - by Thomson when he took into account the existence of the phenomenon of gas ionization in the discharge tube, caused by photons. This phenomenon led to the accumulation of ions on electrodes and the generation of small electric fields. By increasing the gas pressure inside the tube and improving the construction of the electrodes, Thomson discovered the impact of small electric fields on cathode rays, which escaped Hertz's attention. In addition, Thomson gained new knowledge about the phenomena of ionization and the formation of electric charges in space. In conjunction with the conducted experiments on cathode ray deflection, Thomson's experiments pointed to obstacles that need to be removed in order to achieve the expected effect - cathode ray deflection. Thomson's experiments were not only a correction to Hertz's experiments, but turned out to be important in themselves. The phenomenon of gas ionization induced in such a way has become fundamental for the study of electrically active particles in the so-called Willson cloud chamber. Thus, detailed knowledge of the phenomena occurring during the construction and use of a particular cognitive apparatus, says Mayo, made Thomson learn from the mistakes of his predecessors and his own mistakes.

In addition to modifying Kuhn's concept of normal knowledge by extending its scope to experimental practice, Mayo further states that the ability to discover and correct errors through experimentation is already sufficient to trigger or at least initiate scientific revolutions, a thesis that clearly goes beyond the mechanisms of the formation of revolutions described by Kuhn. Mayo sees a good argumentation for her claim in a certain interpretation of Brownian 
motion, which J. Perrin tested in his experiments at the end of the first decade of the 20th century. These experiments have established beyond any doubt that these movements are irregular. These findings, combined with the observational data that the change in particle density distribution is dependent on their height, led Perrin to the conclusion that Brownian particle movements are incompatible with the second law of thermodynamics, and at the same time correspond exactly to the predictions of the hypothetical gas theory. Similarly, Mayo goes on to argue, the experimental research of the radiation of perfectly black bodies, radioactive decay of atoms and photoelectric phenomena forced a revolutionary abandonment of classical physics and laid the foundations, in the first decades of the 20 th century, for a new quantum theory ${ }^{21}$.

\section{CONCLUSIONS: THE ACHIEVEMENTS AND PERSPECTIVES OF THE NEW EXPERIMENTALISM}

Thus the new experimentalism indirectly rejects the accusation that experimental results, dependent on (forced by) theories and paradigms, cannot constitute a legitimate instance for the determination of the truthfulness of empirical theories. The validation of this role as a "referee" for an experiment comes from the constant critical analysis of experimental practice and the use of research equipment (instruments), leading to the elimination of errors, to consideration of counter-problems and modification of problems. Empirical scientific theories can only be modified or altered by experimental research to the extent that the results of the experiments are independent of these theories. One can talk about the rationality of scientific revolutions only if they are forced by experimental results. The perspective of formulating empirical science, all the theories and paradigms of which depend only on speculative assumptions,

21 On the positive and negative role of experimentation, on the understanding of Kuhn's "normal science" as an experiment-based science, on the extension of Kuhn's concept of "scientific revolutions", cf. A. F. Chalmers, Wege der Wissernschaft, op. cit., 162-163. 
is absurd. This kind of science would lose contact with the reality it wants to describe and explain, and experiment is the most characteristic feature of this contact.

The new experimentalism shows how experimental phenomena and results can be justified with the use of numerous and various strategies, e.g.: practical interpretations, formulation and solving of counter-problems, elimination or control of errors, etc., in order to demonstrate their autonomy, their independence from individual or complex theories, their existence. It makes an important contribution to the understanding of the notion of progress in science as increment (expansion) of experimental cognition: the best scientific theories are those that have survived a rigorous experimental test, with the rigorous experimental test being understood as the possibility of rejecting a claim when it is false. It can demonstrate how an experiment can be a criterion for comparing different theories and how it can trigger revolutions in science. Careful analysis of the elements of the experimental procedure serves to check theoretical reasoning and provides a basis for discriminating between what is based on experience and what must be called speculation.

The new experimentalism wants to bring the philosophy of science into the path of facts and proposes a useful correction of some of its, too theoretical, assumptions. However, it does not provide a definitive and holistic answer to the question about the nature of science. This is because an experiment is not absolutely independent of theory. Undoubtedly, it has its own dynamics of development and progress, but similarly, theories also have its own dynamics. The representatives of new experimentalism are right when they claim that it is a mistake to see every experiment as an attempt to answer questions that arise on the basis of a given theory, and not to appreciate at all, or to insufficiently appreciate, the specificity (independent of theory) of the experiment. For example, Galileo was not checking any theory concerning the moons of Jupiter when he directed his telescope to the sky. Since then, many unknown astronomical phenomena have been discovered in a similar way thanks to new instruments and technologies. On the other hand, it is an unde- 
niable fact that theories to a large extent and in a wide scope set the direction for the experimental work and the path to discovering new phenomena. The predictions of Einstein's general theory of relativity were, as we know, the motivation for Eddington's solar eclipse research. Einstein's theoretical contribution to the kinetic theory of gases prompted Perrin to study Brownian motion within a certain range. On the basis of theoretical aspects, the question arose as to whether the frequencies of polarizing changes in dielectric media have a magnetic character, prompting Hertz to start a series of experimental investigations, culminating in the generation of radio waves. The same thing happened with Aragos' discovery of a bright spot in the middle of a darkened glass as a result of an experiment testing Fresnel's wave theory of light.

Whether or not, and regardless of to what extent, an experiment is oriented by some theory, representatives of new experimentalism assess the independence of experimental knowledge from theory. Undoubtedly, Mayo's contribution to such an evaluation of experimental results is significant; her guidance on the use of individual elimination techniques and different kinds of error statistics is particularly valuable. She introduces the concept of an experiment "of the same type", which, on the basis of random control of individual experiments, which are elements of a certain group of them (experiments of the same type), can be assigned a high degree of probability of results. However, the question arises as to how to understand, or how to correctly construct the concept "type of experiment"? Experiments, as we know, can be distinguished in different ways: according to the time they are carried out, according to the place (different laboratories), according to the use of different tools (instruments), etc. A general answer to this question could be a postulate that by "type of experiment" we mean a set of experiments whose essential features are similar. The determination of essential characteristics of an experiment must be, in turn, carried out in relation to the contemporary state of science in a given field; thus, they will change as knowledge is changed, modified, or improved. For example, Galileo conducted a series of experiments from which he 
concluded that acceleration is constant due to gravity. Let us agree that he was also convinced that there was little possibility of data appearing that would be against his thesis. From the present point of view, we know that Galileo's conviction that his thesis is highly probable would be weakened if he carried out his experiments below sea level. If, however, in this kind of experiments, one assumes, as Galileo did, that the tendency for heavy objects to fall is their absolute property, which all objects possess for the sole reason that they are material objects, it is not easy to see that the height above sea level is important in this case and that Galileo's experiments, designed to provide a random control of the acceleration of the fall of bodies, were not representative. The determination of what can be considered as "similar types of experiments" will therefore always depend on a certain theoretical context.

The theoretical context in which the experiment is always located becomes decisive when stating that the results of the experiment go beyond the specific conditions in which they were obtained. One might see this, e.g. in the argumentation of Mayo, who states that the solar eclipse experiments confirm Einstein's theory on gravity. By taking such position, one indirectly concludes that the results of the solar eclipse experiments are exactly the same as those of Newton or Oliver Lodge, who refer to the mechanism associated with the ether as well as any other alternative theory. This is why, in a commentary to the article written by Dyson and Crommelin, Mayo writes that it seems to be the reason why one is forced to resort to Einstein's theory as the only explanation ${ }^{22}$. So, it is not just a matter of showing that until then and in these conditions, the acceptance of Einstein's theory is reasonable, but the main purpose of the argumentation is to make a statement: in fact, other alternative theories do not exist. Naturally, as Chalmers emphasize, Mayo cannot and does not try to exclude the existence of some not yet formulated modification of Newtonian theory, or a theory based on the existence of the ether, which would be able to provide a satisfactory explanation on the

22 Cf. D. Mayo, Error and the Growth of Experimental Knowledge, op. cit., 291. 
results of the solar eclipse experiment. In this context, her advocacy of Einstein's theory, as well as her acceptance of other scientific laws and theories, will be based on the Popperian approach claiming that these survived the rigorous attempts to prove them false, unlike their competing alternatives. The only difference between Mayo's and Popper's followers is that she was able to develop a better version of rigorous inspection, a version in which theoretical considerations play an important role not only in the process of falsification but also in the acceptance of the theorem or theory.

Representatives of the new experimentalism are of the opinion that the experimenters have accurate techniques to reach a credible experimental cognition with this way being relatively independent of the theories in which they work, are guided by or are supported by. As far as the truthfulness of this statement is provided, it seems that the methodological deviations of falsificationism can be corrected and at the same time it can be acknowledged as the cumulative aspect of scientific advance understood as the growth (enlargement) of trustworthy experimental cognition. However, if the theoretical assumptions and elements are assigned an important role in the progress and obtaining experiment results, one has to agree with the existence of a certain range of errors in experimental cognition. The new experimentalism cannot in this case indicate how to eliminate theories or theoretical constructions from science (scientific cognition). In the context of these considerations, however, it may be purposeful to note that an important factor in determining the accuracy of Newtonian mechanics in the area of interplanetary travel was mass which, if not taken into account - at a given speed - was an important counter-argument to Newton's theory of relativity. Undoubtedly, theories have "their own life" in science. The principles of quantum mechanics widely used in science, for example, to improve the electron microscope or to obtain energy, are much more than just a generalization of specific experiments. Thus, the questions arise: what kind of a "peculiar life of theory" is it and what is its connection with an experiment? Some representatives of the new experimentalism would like to draw a sharp demarcation line 
between well-established experimental cognition on the one hand and theories on the other. Mayo seems to belong to this group when she differentiates between the general theory of relativity and the theory of gravitation experimentally proven by Eddington. Others do not attempt to make this kind of distinction, believing that only experimental laws provide an opportunity to formulate verifiable statements about the world. On the other hand, they consider that theories are some kind of organizational and heuristic structures and not statements about the real world ${ }^{23}$.

Many theorists of science and methodologists agree with the claim that the value of a theory is manifested by the extent to which it can withstand radically strict verification. However, there is a considerable number of theories (theorems) in science that undoubtedly do not meet this requirement. In these cases, a significant correspondence between theory and observation can also be established, but only if the failure to meet the formulated postulate is not an argument against these theories.

The normal practice of the empirical sciences is, among other things, that from theories and various, sometimes even questionable assumptions, some kind of predictions are derived. Experimental confirmation of these predictions is considered to be an important confirmation of theories. The reason for the negative result of the confirmation of the predictions, i.e., their contradiction, may be either in the theory itself, or in the auxiliary assumptions, or in the theory and auxiliary assumptions at the same time. Hence, not every falsification of a prediction is an argument for rejecting a theory. As a consequence, it may seem that a verification in which some predictions appear which are contrary to experience, is not strong (radical) enough, but such a theory can obtain significant reinforcement with other confirmations. The following example can be an illustration of this problem, in which N. Thomason was very interested ${ }^{24}$. Coper-

23 On the successes and prospects of new experimentalism cf. A. F. Chalmers, Wege der Wissernschaft, op. cit., 164-167.

24 Cf. N. Thomason, The power of ARCHED Hypotheses: Feyerabend's Galileo as a Closet Rationalist, British Journal of the Philosophy of Science 45(1994), 255-264; Idem, 1543 
nicus' theory assumes that the planet Venus appears in the form of phases which in a specific way correspond to, and correlate with the phases of the Moon. On this basis, it was assumed that Venus is not permeable to light. Both Copernicus and Galileo considered this assumption to be an open issue. Galileo could use his telescope to confirm the occurrence of phases of the planet Venus according to the assumptions of Copernican theory. Combined with the assumption that Venus is not permeable to light, the result of telescopic observations would be a strong confirmation of the theory and auxiliary assumption. If the phases of the planet Venus could not be observed, the reasons for this could be found both in the theory and in the auxiliary assumption. Such an observation procedure would therefore not be a strong test of the Copernican system.

A similar and relatively frequent situation is encountered when the observations which examine a given theory are ambiguous. In this case, the compatibility of theoretical predictions with the presented observations can confirm both the theory and interpretation of the observations, while a lack of compatibility only indicates the need for some changes or modifications. An example is the use of an electron microscope to observe the dislocation (shift) of atoms in crystal structures. The occurrence of these dislocations, i.e. deviations from the regular arrangement of atoms in crystalline materials, was theoretically predicted in the 1830s. The dislocations provide the crystals with their characteristic durability, extensibility and plasticity. If there was perfect order (perfect regularity) in the crystalline structures, then too much tension of forces would form in the crystal lattice, and these would destroy the known durability and known shapes of crystalline bodies. An improved electronic microscope allowed, twenty years later, to observe the crystalline lattice and dislocations, but it was not yet good enough (the theory of interaction between electrons and observed crystalline samples was still imperfect) to definitely verify the theoretical predictions. It was

- The Years That Copernicus Didn't Predict the Phases of Venus, in: 1543 and All That, ed. A. Corones, G. Freelan, Reidel, Dordrecht 1998. 
not until 1956 that J. Menter ${ }^{25}$ and P. B. Hirsch ${ }^{26}$ constructed an electron microscope that was perfect enough to identify dislocations well. Some ways of the proper interpretation of the complex of microelectronic photographs proposed by them strongly resemble the techniques proposed by the representatives of the new experimentalism when identifying the results of an experiment. This is how, for example, the consequences of practical interventions, such as bending of the crystals, have been observed and determined, which was consistent with the pictures. The pictures showed the crystal lattice and the phenomena of occurrence of such different physical processes as X-ray radiation and electron diffraction. The extent to which these phenomena were compatible leads to the conclusion that in this case theory and observation confirm each other. Menter, for example, used Abbe's theory of microscope construction to take pictures of crystal lattices. He considers the essential correspondence between the prediction and the received images to be a confirmation of both his theory and his interpretation of the images, as an image of the crystal lattice. Hirsch also used his observations which indicate that dislocations are arranged according to the assumptions of current theories to consider them as the confirmation of both the theory and the fact that the images are an image of dislocations.

In all these cases, the conformity of the theory with predictions is an important confirmation of a theory. In other cases, experimental situations were so unspecified and incomprehensible that they allowed for other causes of failure than those that were close to the tested dislocation theory. One can expect, as Chalmers states ${ }^{27}$, that the described example of behaviour is the norm of experimental science in general. The methodological characteristics of strong verification postulated by Mayo can be used in the above-mentioned examples. The fundamental question is, is it likely that a false theory can get this

25 J. Menter, The Direct Study by Electron Microscopy of Crystal Lattices and Their Imperfections, Proceedings of the Royal Society, A 236(1956), 119-135.

26 P. B. Hirsch, R. W. Horne, M. J. Whelan, Direct Observation of the Arrangements and Motions of Dislocations in Aluminium, Philosophical Magazine 1(1956), 677-684.

27 A. F. Chalmers, Wege der Wissernschaft, op. cit., 168-169. 
kind of strong experimental confirmation? In both cases discussed, Copernicus' theory and the theory of dislocation, a positive answer is extremely unlikely. The methodological postulate of the future, derived from the considerations presented, could be: all the theories concerning the empirical world should be confirmed by the encounter of theoretical predictions with the widest possible range of strong experimental verification (strong observation). The conception proposed by the representatives of the new experimentalism, especially Mayo's proposals for radical checking, are well in harmony with the modern scientific practice ${ }^{28}$.

\section{BIBLIOGRAPHY}

Ackermann R., The New Experimentalism, British Journal of the Philosophy of Science 40(1989), 185-190.

Buchwald J., The Creation of Scientific Effects, University of Chicago Press, Chicago 1989.

Chalmers A. F., Wege der Wissernschaft (Einfuerung in die Wissenschaftstheorie), Springer - Verlag, Berlin - Heidelberg 2001.

Filozofia a nauka, ed. Z. Cackowski, Ossolineum - PAN, Wrocław - Warszawa Kraków - Gdańsk - Łódź 1987.

Franklin A., Experiment, Right or Wrong, Cambridge University Press, Cambridge 1990.

Franklin A., The Neglect of Experiment, Cambridge University Press, Cambridge 1986.

Galison P., How Experiments End, University of Chicago Press, Chicago 1987.

Galison P., Image and Logic: A Material Culture of Physics, University of Chicago Press, Chicago 1997.

28 The group of representatives of new experimentalism, apart from the said authors, also includes A. Franklin, The Neglect of Experiment, Cambridge University Press, Cambridge 1986; Idem, Experiment, Right or Wrong, Cambridge University Press, Cambridge 1990; P. Galison, How Experiments End, University of Chicago Press, Chicago 1987; Idem, Image and Logic: A Material Culture of Physics, University of Chicago Press, Chicago 1997; D. Gooding, Experiment and the Making of Meaning: Human Agency in Scientific Observation and Experiment, Kluwer, Dordrecht 1990. 
Gooding D., Experiment and the Making of Meaning: Human Agency in Scientific Observation and Experiment, Kluwer, Dordrecht 1990.

Hacking I., Einfuerung in die Philosophie der Naturwissenschaften, Philipp Reclam, Stuttgard 1996.

Hertz H., Gesammelte Werke, vol. II: Untersuchungen ueber die Ausbreitung der elektrischen Kraft, Bahrt, Leipzig 1894.

Hirsch P. B., Horne R. W., Whelan M. J., Direct Observation of the Arrangements and Motions of Dislocations in Aluminium, Philosophical Magazine 1(1956), 677-684.

Lakatos I., Falsifikation und die Methodologie wissenschaftlicher Forschungsprogramme, in: Kritik und Erkenntnisfortschritt, ed. I. Lakatos, A. Musgrave, Vieweg, Wiesbaden 1974.

Maxwell J. C., Illustrations of the Dynamical Theory of Gases, in: The Scientific Papers of James Clerk Maxwell, 2 volumes, ed. W. D. Niven, Dover, New York 1965.

Maxwell J. C., The Kinetic Theory of Gases, Nature 16(1877), 245-246.

Mayo D., Error and the Growth of Experimental Knowledge, University of Chicago Press, Chicago 1996.

Menter J., The Direct Study by Electron Microscopy of Crystal Lattices and Their Imperfections, Proceedings of the Royal Society, A 236(1956), 119-135.

Thomason N., 1543 - The Years That Copernicus Didn't Predict the Phases of Venus, in: 1543 and All That, ed. A. Corones, G. Freelan, Reidel, Dordrecht 1998.

Thomason N., The power of ARCHED Hypotheses: Feyerabend's Galileo as a Closet Rationalist, British Journal of the Philosophy of Science 45(1994), 255-264.

Thomson W., Tait P. G., Handbuch der theoretischen Physik, Vieweg, Braunschweig 1879.

Mieczysław Bombik

DOI: $10.21697 /$ spch.2020.56.S2.02 